\title{
Schemes for robust quantum computation with polar molecules
}

\author{
S. F. Yelin, ${ }^{1,2}$ K. Kirby, ${ }^{2}$ and Robin Côté ${ }^{1,2}$ \\ ${ }^{1}$ Department of Physics, University of Connecticut, Storrs, CT 06269 \\ ${ }^{2}$ ITAMP, Harvard-Smithsonian Center for Astrophysics, Cambridge, MA 02138
}

(Dated: August 1, 2018)

\begin{abstract}
We propose to use a new platform - ultracold polar molecules - for quantum computing with switchable interactions. The on/off switch is accomplished by selective excitation of one of the $|0\rangle$ or $|1\rangle$ qubits - long-lived molecular states - to an "excited" molecular state with a considerably different dipole moment. We describe various schemes based on this switching of dipolar interactions where the selective excitation between ground and excited states is accomplished via optical, microwave, or electric fields. We also generalize the schemes to take advantage of the dipole blockade mechanism when dipolar interactions are very strong. These schemes can be realized in several recently proposed architectures.
\end{abstract}

Quantum computing is one of the most rapidly developing areas in physics today. For certain tasks, quantum computers have significant potential to outperform classical computers [1]. Several platforms are being investigated to implement these ideas, e.g., using atomic, molecular and optical, condensed matter, and other systems. A key challenge in all of these approaches is to identify strong and controllable interactions that would allow for the creation of fast quantum operations with minimal decoherence.

Quantum information processing makes use of quantum superposition in which the fundamental piece of information, called a qubit, consists of a superposition of quantum states, denoted $|0\rangle$ and $|1\rangle$. The building blocks of a quantum computer consist of "gate" operations, in which a coherent change in the state of one qubit can be brought about through a carefully controlled interaction with another qubit, and the result is dependent on the state of the second qubit. In order to implement reversible quantum logic operations it is essential to address these quantum states coherently.

Of the various platforms proposed to implement quantum computers, trapped ions and neutral atoms are especially attractive [1]. Trapped ions [2] exhibit strong interactions and are relatively easy to control, while neutral atoms [3] have long coherence times and techniques to cool and trap them are well developed. Polar molecules represent a new platform that might incorporate the biggest advantages of both atoms and ions and even bridge the gap with condensed matter physics approaches (e.g. molecule-chips 4] or microtraps connected to superconducting wires [5]). They have long coherence times like neutral atoms, and strong interactions like trapped ions. However, contrary to ions, the interactions can be made "switchable," a feature which would help to simplify phase gates and minimize decoherence. Advances in cooling [6] and storing [7] techniques for molecules are beginning to make possible the required accurate manipulation of single molecules.

In this Letter, we investigate the implementation of universal two-qubit logic gates in realistic systems, using ultracold polar molecules. As opposed to other schemes using polar molecules [8], such as vibrational eigenstates [9] and optimal control [10], our approach is based on the ability to "switch" on and off dipole interactions between polar molecules. For this, we use the fact that all heteronuclear molecules have (a) different dipole moments depending on their electronic, vibrational, or rotational states, and (b) zero expectation value for the dipole moment in the $\mathrm{N}=0$ rotational state.

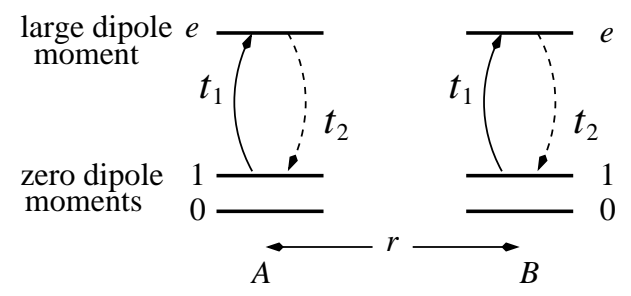

FIG. 1: Phase gate: two molecules $A$ and $B$ separated by $r$ are prepared in a superposition of states $|0\rangle$ and $|1\rangle$. At $t_{1}=0$, we excite $|1\rangle$ of both into $|e\rangle$ : both interact via dipoledipole interactions, and acquire a phase $\phi$. At time $t_{2}=\tau$ such that $\phi=\pi$, we stimulate coherently both $|e\rangle$ back to $|1\rangle$.

We first describe the generic setup to obtain a phase gate, or universal two-qubit operation, in Fig.11 We assume that the molecules are individually addressable by optical or microwave fields, and choose $|0\rangle$ and $|1\rangle$ as, for example, hyperfine states, in part of a zero-dipolemoment manifold in a level with a long coherence time and $|e\rangle$ is a metastable state in a large-dipole-moment manifold. Single-qubit rotations can be accomplished with optical or microwave fields. The initial states of two individual sites $A$ and $B$ can be prepared in a superposition state, e.g. using $\pi / 2$ Raman pulses. A oneor two-photon transition couples $|1\rangle$ and $|e\rangle$ coherently, but not $|0\rangle$ and $|e\rangle$. This can always be accomplished by either polarization or frequency selection. The molecules interact via a dipole-dipole interaction only if both are in the $|e\rangle$ state, and acquire a phase $\phi(t)$. After a time $t=\tau$ such that $\phi=\pi$, we coherently stimulate the states 
$|e\rangle$ back to $|1\rangle$. This can be summarized by

$$
\begin{aligned}
& |00\rangle \stackrel{\pi-\text { pulse }}{\longrightarrow}|00\rangle \stackrel{\text { dip-dip }}{\longrightarrow}|00\rangle \stackrel{\pi-\text { pulse }}{\longrightarrow}|00\rangle \\
& |01\rangle \longrightarrow|0 e\rangle \longrightarrow|0 e\rangle \longrightarrow|01\rangle \\
& |10\rangle \longrightarrow|e 0\rangle \longrightarrow|e 0\rangle \longrightarrow|10\rangle \\
& |11\rangle \longrightarrow|e e\rangle \longrightarrow-|e e\rangle \longrightarrow-|11\rangle
\end{aligned}
$$

The resulting transformation corresponds to a phase gate. We desire the wave function, expressed as $e^{-i E \Delta t / \hbar}$, to acquire a phase shift of $\pi$, thus becoming $e^{-i \pi}$, as $\mathrm{A}$ and $\mathrm{B}$ experience dipole-dipole interaction in state $|e\rangle$. The $\pi$-phase shift produced in the time $\tau$ between the exciting and de-exciting $\pi$-pulses is given by

$$
\phi=\pi=\frac{1}{\hbar} \int_{0}^{\tau} d \tau^{\prime} \frac{d^{2}}{r^{3}}\left(3 \cos ^{2} \theta-1\right) \rho_{e}^{2}\left(\tau^{\prime}\right),
$$

where $d$ and $\rho_{e}$ are the dipole moment and fractional population in the excited state, $r$ the distance between molecules $A$ and $B$, and $\theta$ the angle between the dipole moments. This formulation allows for finite excitation and de-excitation times and imperfect $\pi$-pulses.
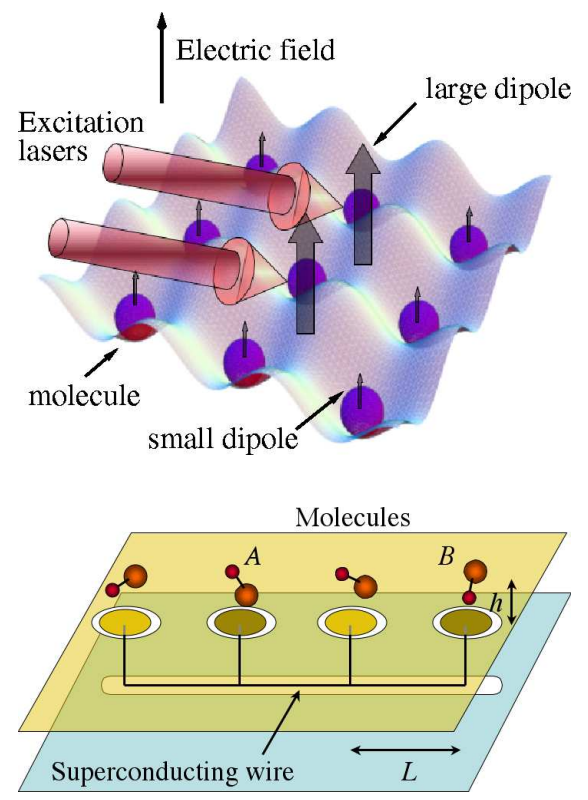

FIG. 2: Setups: (Top) molecules individually addressable by lasers are stored in an optical lattice, (Bottom) superconducting wires are used to "deliver" the interaction. In both, molecules are selectively excited, and interact only if both are in $|e\rangle$.

To implement the scheme, molecules are stored in a $1 \mathrm{D}$ or $2 \mathrm{D}$ array so that their dipole moments could be aligned by an electric field perpendicular to the array. We assume the full development of the storage and addressing capabilities of two recently proposed architectures (see Fig. 2). The first is an optical lattice with a lattice spacing of about $1 \mu \mathrm{m}$, as suggested by DeMille [8]. Using a DC field for dipole alignment during trapping naturally allows the repulsive dipole-dipole interaction to aide with homogeneous distribution in the lattice. In this case, addressing single qubits can be accomplished by either using the inhomogeneous DC electric fields proposed by DeMille to create individualized transition frequencies, or by individual addressing with light in the visible part of the frequency spectrum. The second architecture is based on a "strip wire" architecture, as suggested at Yale and Harvard [5]; molecules sit on their own small microwave traps which also serve for addressing, and are connected via a superconducting wire that allows for long-range dipole-dipole interaction, effectively replacing the $1 / r^{3}$ term in Eq. (1) with $1 / h^{2} r$, where $h$ is the distance of the molecules from the wire. Here, all the fields need to be in the microwave range.

We now describe three possible setups utilizing variations of our switchable phase-gate scheme. The first system is based on carbon monoxide (CO). As far as dipolar molecules are concerned, $\mathrm{CO}$ is an anomaly; while its electronic ground state $X^{1} \Sigma^{+}$has a very small dipole moment ( $\mu \approx 0.1 \mathrm{D}$ in the vibrational ground state which is expected to be the easiest to trap), there exists a very long-lived $\left(\tau_{\text {life }} \approx 10-1000 \mathrm{~ms}\right)$ excited electronic state $a^{3} \Pi$ with a large dipole moment, $\mu \approx 1.5 \mathrm{D}$. As " 0 " and "1," we choose, for example, two hyperfine states of $X^{1} \Sigma^{+}, v=0, N=0$ of ${ }^{13} \mathrm{CO}$ [1]. With a hyperfine splitting of about $1 \mathrm{MHz}$, selective excitation from $|1\rangle$ to $|e\rangle$ is possible. The transition frequency between $X^{1} \Sigma^{+}$ and $a^{3} \Pi$ is in the UV (about $48,000 \mathrm{~cm}^{-1}$ ), and the optical lattice architecture would be the ideal choice. With a coherence time in an optical lattice of a few seconds [8] and possibly much less [12 and a necessary dipoledipole interaction time of several milliseconds, there can be about $10^{3}$ operations. The scheme, however, is very straightforward, the techniques are in place or nearly so, and $\mathrm{CO}$ is a very well studied molecule [13].

A more common situation can be found in molecules such as alkali hydrides or mixed alkali dimers, e.g. LiH or LiCs. These molecules have large permanent dipole moments $\mu$ (as large as $7 \mathrm{D}$ ) in their ground electronic state $X^{1} \Sigma^{+}$(for $|0\rangle$ and $|1\rangle$ ), and a metastable electronic state $a^{3} \Sigma^{+}$(for $|e\rangle$ ) for which the potential well is located at large nuclear separation and supports at least one bound state; in most cases, these triplet states have permanent dipole moments close to zero. These properties can be used to implement a scheme in all important points similar to the $\mathrm{CO}$ scheme, except for three details. First, the phase gate would be "inverted", i.e. $|00\rangle \rightarrow-|00\rangle$, $|01\rangle \rightarrow|01\rangle,|10\rangle \rightarrow|10\rangle$, and $|11\rangle \rightarrow|11\rangle$. Second, it requires the molecules to be stored, with the help of an aligning DC electric field, in the large-dipole state which would most likely lead to seriously shortened coherence times. In addition, the interaction would happen for all molecules, not just the two we wish to be coupled by a phase gate. However, this can be mitigated by switching on an aligning DC field only during interaction times, 
and for exactly a $2 \pi$ phase shift.

For any molecule in a pure $N=0$ rotational state, the expectation value of its dipole moment is zero. Such states can acquire a dipole moment by the application of an electric field that mixes $N=0$ and $N=1$ states. So, by adding together the $2 \pi$ phase shift using a DC field and the "negative" $\pi$ phase shift for the molecules in the $|e\rangle$ state, the phase gate is given by

\begin{tabular}{|c|c|c|c|c|c|c|c|}
\hline 00$\rangle \stackrel{\text { exc+Dc }}{\longrightarrow}$ & $|00\rangle$ & $\stackrel{\pi}{\longrightarrow}$ & $-|00\rangle$ & $\stackrel{\text { de-exc }}{\longrightarrow}$ & $-|00\rangle$ & $\stackrel{D C}{\longrightarrow}$ & $|00\rangle$ \\
\hline 1) & $|0 e\rangle$ & $\longrightarrow$ & $|0 e\rangle$ & $\longrightarrow$ & $|01\rangle$ & & $-|01\rangle$ \\
\hline$\rightarrow$ & $|e 0\rangle$ & $\longrightarrow$ & $|e 0\rangle$ & $\longrightarrow$ & $|10\rangle$ & $\longrightarrow$ & $-|10\rangle$ \\
\hline 1) & $|e e\rangle$ & $\longrightarrow$ & $|e e\rangle$ & $\longrightarrow$ & $|11\rangle$ & $\longrightarrow$ & $-|11\rangle$ \\
\hline
\end{tabular}

Note that the scheme described for $\mathrm{CO}$ could be adapted for these molecules by using two different vibrational states of $a^{3} \Sigma^{+}$as $|0\rangle$ and $|1\rangle$, and a low-level vibrational state of $X^{1} \Sigma^{+}$as $|e\rangle$.

The last setup we propose here is the "rotational scheme". It utilizes the fact that in the rotational ground state $N=0$ the dipole moment $\mu$ is, in fact, zero. We choose for all states the electronic and vibrational ground state. While $|0\rangle,|1\rangle$ are also in the rotational ground state $N=0,|e\rangle$ is the superposition of neighboring rotational states $|e\rangle=\left|e_{1}\right\rangle+\left|e_{2}\right\rangle$, as shown in Fig. [19]. Because both $|0\rangle$ and $|1\rangle$ are in the absolute ground state with exactly zero dipole moment, this system has several advantages: maximum coherence time, ease of storage, and no residual dipole-dipole interaction. Moreover, any polar molecule can be used with this scheme, as long as it has at least two hyperfine states. One interesting choice would be $\mathrm{NaCl}$ with a dipole moment of up to $10 \mathrm{D}$.

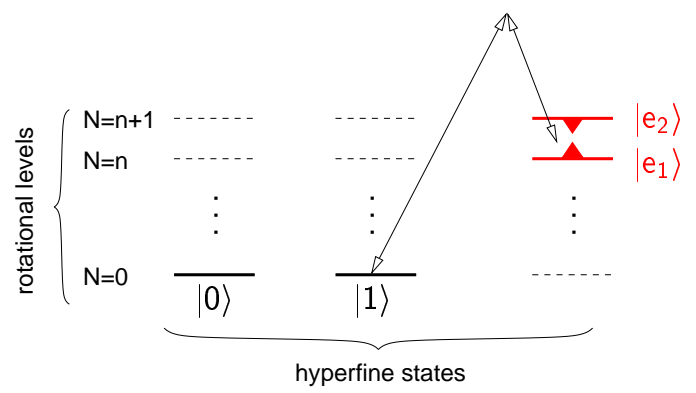

FIG. 3: Example of level system for "rotational scheme": all states are part of the electronic and vibrational ground state. $|0\rangle$ and $|1\rangle$ are in different hyperfine states. $|0\rangle,|1\rangle$ are $|N=0\rangle$ states, $|e\rangle \propto\left|e_{1}\right\rangle+\left|e_{2}\right\rangle$ is a superposition between two adjacent rotational states $|N=n\rangle$ and $|N=n+1\rangle$.

Given the fact that rotational levels are spaced in the $\mathrm{GHz}$ range and thus only low-frequency photons are required, this scheme is suitable for both the optical lattice and the superconducting wire architectures; for a dipole moment $\mu=10 \mathrm{D}, r=10 \mu \mathrm{m}$, and $h=0.1 \mu \mathrm{m}$, the necessary interaction time is of the order of $3 \mu \mathrm{s}$. With a coherence time of the order of $100 \mathrm{~ms}-1 \mathrm{~s}$, this setup would thus allow for $10^{5}-10^{6}$ operations.
If the sites can be addressed individually and the dipole-dipole interactions are very strong, the previous schemes could take advantage of the so-called dipole blockade mechanism. This mechanism has been introduced for quantum information processing with Rydberg atoms in [15], and generalized to mesoscopic ensembles [16]. A variant of the dipole blockade based on the strong van der Waals interactions between Rydberg atoms, known as the $v d W$ blockade, has been explored experimentally [17]. The underlying principle goes as follows: strong Rydberg-Rydberg interactions shift the energy levels, so that one atom can be resonantly excited into a Rydberg state, but additional Rydberg excitations are prevented by the large shifts.

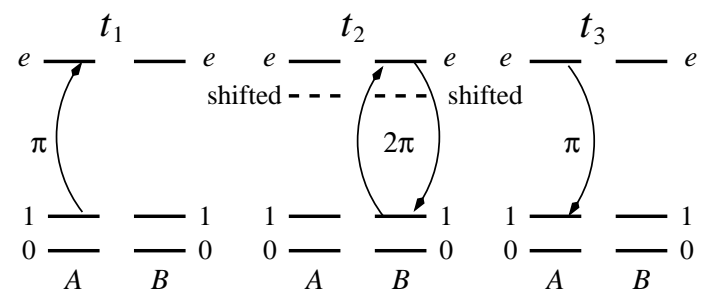

FIG. 4: Principle of the dipole blockade (see text).

The same idea can be applied to polar molecules. If the dipole-dipole interaction is strong enough, 1.e. larger than the bandwidth of the excitation laser, the doublyexcited state corresponding to $|e e\rangle$ will be shifted out of resonance and never excited. If both sites $A$ and $B$ are addressable individually, the ability to drive a $2 \pi$ transition in site $B$ depends on whether site $A$ is excited (see Fig. (4). At $t_{1}$, we apply a $\pi$ pulse to molecule $A$ and populate the state $|e\rangle$. At $t_{2}$ we apply a second pulse $(2 \pi)$ to molecule $B$ : if $A$ is already in $|e\rangle_{A}$, the dipole-dipole interaction shifts the state $|e\rangle_{B}$, the photon is off-resonance, hence no transition. If $A$ is not in $|e\rangle_{A}, B$ acquires a phase of $\pi$ after the process. At $t_{3}$, we de-excite $A$ with another $\pi$ pulse; in summary

\begin{tabular}{|c|c|c|c|c|c|c|}
\hline \multicolumn{3}{|c|}{$t_{1}$} & $t_{2}$ & & $t_{3}$ & \\
\hline$|00\rangle$ & & $|00\rangle$ & & $|00\rangle$ & & $|00\rangle$ \\
\hline$|01\rangle$ & & $|01\rangle$ & $\longrightarrow$ & $-|01\rangle$ & & $-|01\rangle$ \\
\hline$|10\rangle$ & $\longrightarrow$ & $i|e 0\rangle$ & & $i|e 0\rangle$ & $\longrightarrow$ & $-|10\rangle$ \\
\hline$|11\rangle$ & $\longrightarrow$ & $i|e 1\rangle$ & $x \rightarrow$ & $i|e 1\rangle$ & $\longrightarrow$ & $-|11\rangle$ \\
\hline
\end{tabular}

This scheme is robust with respect to the separation between the molecules; as long as the excitation is blockaded, the exact separation is not important.

A key operation at the end of several qubit operations is the readout of the quantum registers. Several approaches could be employed with polar molecules. For example, selective ionization of one of the states ( 0 or 1 ) and the detection of molecular ions can be readily accomplished. However, this is a destructive method, since the molecule is lost after the readout, and the site would need to be refilled. A different method uses a "cycling" 
fluorescent transition in which the molecules decay after irradiation directly back into the state from which they came. Although this might be more difficult for molecules than for atoms because of the large number of molecular levels, it offers the advantage of being "non-destructive." Another approach based on recent work on evanescentwave mirrors for polar molecules might yield promising results [18]; while "0" would stick to the wall, "1" could be reflected. Because reflection takes place far away from the surface of the mirror, it might help to minimize decoherence due to shorter range interactions with the surface. The schemes described above may suffer from various sources of error. As our schemes rely only on internal molecular states, there is a possibility that some of the molecules may be translationally "hot." If molecules are not in the motional ground state of the trap, there can be considerable uncertainty and variation in the separation between molecules, which can affect the exact phase. For example, during a $\sim 1 \mu$ s gate time, the motion of RbCs molecules at $10 \mu \mathrm{K}$ can lead to $\sim 3 \%$ variation in the phase. We can control and reduce such error, e.g., using molecules with larger dipole moments, larger separations, shorter gate times, or lower temperatures. Note that decoherence and uncertainty due to molecular motion can be completely eliminated using dipole blockade, leading to higher fidelity [16].

Finally, several technical issues may affect the implementation of our schemes, such as turning on and off electric fields, misalignments of dipoles, decoherence in an optical lattice (e.g., incoherent photon scattering or ionization), or imperfect excitation pulses; these can be overcome, e.g., by trapping molecules with evanescentwave mirrors, using Stimulated Raman Adiabatic Passage (STIRAP) or chirped pulses. Other physical effects such as DC and AC Stark mixing, spontaneous decay of metastable molecular states, or rovibrational quenching if sites contain more than one molecule, can be avoided by a judicious choice of molecules and states, and careful loading of sites.

In summary, we propose a new platform that combines the advantages of both neutral atoms, such as long coherence times, and trapped ions, such as strong interactions. Contrary to ions, the interactions can be made "switchable," a feature helping to simplify phase gates considerably and thus to minimize decoherence. Using these techniques, up to $10^{6}$ operations should be obtainable in the available coherence time. Finally, the possibility exists to bridge the gap with condensed matter devices, using polar molecules instead of quantum dots in circuits with superconducting wires that convey the interaction.

We would like to thank D. DeMille, J. Doyle, M. Lukin, and D. Petrov for fruitful discussions. Partial funding from the National Science Foundation and the Research Corporation is gratefully acknowledged.
[1] http://qist.lanl.gov/qcomp_map.shtml

[2] J.I. Cirac and P. Zoller, Phys. Rev. Lett. 74, 4091 (1995); C. Monroe et al., Phys. Rev. Lett. 75, 4714 (1995); B.E. King et al., Phys. Rev. Lett. 81, 1525 (1998).

[3] D. Jaksch et al., Phys. Rev. Lett. 82, 1975 (1999); O. Mandel et al., Nature 425, 937 (2003).

[4] M. Vengalattore, R.S. Conroy, and M.G. Prentiss, Phys. Rev. Lett. 92, 183001 (2004); S. Groth et al., Appl. Phys. Lett. 85, 2980 (2004); C.D.J. Sinclair et al., Phys. Rev. A 72, 031603(R) (2005); J. Janis, M. Banks, and N.P. Bigelow, Phys. Rev. A 71, 013422 (2005).

[5] A. Wallraff et al., Phys. Rev. Lett. 95, 060501 (2005); A.S. Sorensen et al., Phys. Rev. Lett. 92, 063601 (2004).

[6] K. Maussang et al., Phys. Rev. Lett 94, 123002, (2005); A.J. Kerman et al., Phys. Rev. Lett. 92, 153001 (2004); E. Hodby et al., Phys. Rev. Lett. 94, 120402 (2005); M.R. Tarbutt et al., Phys. Rev. Lett. 92, 173002, (2004); D. Wang et al., Phys. Rev. Lett. 93, 243005 (2004); C. Haimberger et al., Phys. Rev. A 70, 021402(R) (2004).

[7] J.G.E. Harris et al., Rev. Sci. Instrum. 75, 17 (2004); J. van Veldhoven, H.L. Bethlem, and G. Meijer, Phys. Rev. Lett. 94, 083001 (2005); S.Y.T. van de Meerakker et al., Phys. Rev. Lett. 94, 023004 (2005); T. Junglen et al., Phys. Rev. Lett. 92, 223001 (2004).

[8] D. DeMille, Phys. Rev. Lett. 88, 067901 (2002).

[9] U. Troppmann, C.M. Tesch, R. de Vivie-Riedle, Chem. Phys. Lett. 378, 273 (2003); D. Babikov, J. Chem. Phys. 121, 7577 (2004).

[10] J.P. Palao and R. Kosloff, Phys. Rev. Lett. 89, 188301 (2002).

[11] Note that, since ${ }^{12} \mathrm{CO}$ has a zero nuclear spin, isotopic ${ }^{13} \mathrm{CO}$ would have to be used.

[12] The residual dipole moment in the ground state may lead to much more decoherence than is given by the lattice.

[13] R.H. Gammon et al., J. Chem. Phys. 54, 2136 (1971); R.T. Jongma et al., Chem. Phys. Lett. 270, 304 (1997); H.L. Bethlem et al., Phys. Rev. Lett. 88, 133003 (2002).

[14] It is important to ensure that no single-photon transitions are possible between $|0\rangle$ or $|1\rangle$ and $|e\rangle$. Otherwise a virtual photon exchange could happen between the "active" qubits (in $|e\rangle$ ) and any neighboring molecule, resulting in "diffusion" of the excitation.

[15] D. Jaksch et al., Phys. Rev. Lett. 85, 2208 (2000).

[16] M.D. Lukin et al., Phys. Rev. Lett. 87, 037901-1 (2001).

[17] D. Tong et al., Phys. Rev. Lett. 93, 063001 (2004).

[18] S. Kallush, B. Segev, and R. Côté, Phys. Rev. Lett. 95, 163005 (2005); S. Kallush, B. Segev, and R. Côté, Eur. Phys. J. D 35, 3 (2005).

[19] $|e\rangle$ is subject to the following restrictions: (1) $|e\rangle$ has to be coupled by a two- or more photon transition to $|1\rangle$ and this photon combination must not couple $|0\rangle$; (2) in order to have a non-zero dipole moment there has to be an electric dipole allowed transition between $\left|e_{1}\right\rangle$ and $\left|e_{2}\right\rangle$; (3) there must not be an electric dipole allowed transition between $\left|e_{1 / 2}\right\rangle$ and either $|0\rangle$ or $|1\rangle$, since otherwise spontaneous diffusion of the excitation in the molecular sample could occur. 